\title{
Physical Activity Breaks and Facilities in US Secondary Schools
}

Nancy E. Hood, PhD, MPha Natalie Colabianchi, PhD ${ }^{b}$ Yvonne M. Terry-McElrath, MSAc Patrick M. O'malley, PhD ${ }^{d}$ Lloyd D. Johnston, PhD $^{\mathrm{e}}$

ABSTRACT

BACKGROUND: Research on physical activity breaks and facilities (indoor and outdoor) in secondary schools is relatively limited.

METHODS: School administrators and students in nationally representative samples of 8th (middle school) and 10th/12th grade (high school) students were surveyed annually from 2008-2009 to 2011-2012. School administrators reported information about physical activity breaks and facilities. Students self-reported height, weight, and physical activity.

RESULTS: The prevalence of physical activity breaks and indoor and outdoor facilities (dichotomized by median split) differed significantly by region of the country, school size, student race/ethnicity, and school socioeconomic status (SES). Breaks were associated with lower odds of overweight (adjusted odds ratio $[A O R]=0.91,95 \%$ confidence interval [CI]: $0.83-1.00$ ) and obesity $(A O R=0.86,95 \% \mathrm{Cl}: 0.75-0.99)$ among middle school students. Among low-SES middle school students and schools, higher indoor facilities were associated with lower rates of overweight and obesity. Among high school students, higher indoor and outdoor facilities were associated with $19-42 \%$ higher odds of moderate-to-vigorous physical activity.

CONCLUSIONS: Physical activity breaks and school facilities may help to address high rates of overweight/obesity and low physical activity levels among secondary students, especially lower-SES students. Students in all schools should have equal access to these resources.

Keywords: physical activity; environment; policy; adolescent.

Citation: Hood NE, Colabianchi N, Terry-McElrath YM, O'Malley PM, Johnston LD. Physical activity breaks and facilities in US secondary schools. J Sch Health. 2014; 84: 697-705.

Received on December 2, 2013

Accepted on February 17, 2014

Chools can play an important role in addressing $\mathcal{S}$ increasing overweight and obesity by creating environments that make it easier for students to be active. $^{1}$ It is recommended that youth achieve at least 60 minutes of moderate or vigorous physical activity every day, ${ }^{2}$ approximately half of which should occur at school. ${ }^{3}$ Evidence-based physical education (PE) classes have long been recognized as an effective strategy for increasing students' physical activity at school ${ }^{4}$ and should be required daily for all students. ${ }^{3}$ However, because curricular demands may prevent schools from offering the recommended amount of physical activity during PE classes alone, schools should consider creating other opportunities for students to be active during the day. ${ }^{5,6}$

Recess and physical activity breaks are both broadly defined as opportunities during the school day for students to be physically active outside of PE classes. ${ }^{7,8}$ Additional recess periods or inexpensive interventions to increase activity levels during breaks in the school day have been shown to increase physical activity and fitness above baseline levels among elementary school students, including several with strong study designs, ${ }^{5,9,10}$ but are largely unstudied

aResearch Fellow, (nhood@cpoms.org), Institute for Social Research, University of Michigan, 426 Thompson Street, Room 2346, Ann Arbor, Ml 48106.

b Assistant Research Professor, (colabian@umich.edu), Institute for Social Research, University of Michigan, 426 Thompson Street, Room 2346, Ann Arbor, MI 48106.

'Research Associate, (yterry@umich.edu), Institute for Social Research, University of Michigan, 426 Thompson Street, Room 2344, Ann Arbor, Ml 48106.

dResearch Professor, (pomalley@umich.edu), Institute for Social Research, University of Michigan, 426 Thompson Street, Room 2320, Ann Arbor, Ml 48106.

eAngus Campbell Collegiate Research Professor and University Distinguished Senior Research Scientist, (lloydj@umich.edu), Institute for Social Research, University of Michigan, 426 Thompson Street, Room 2324, Ann Arbor, Ml 48106.

Address correspondence to: Natalie Colabianchi, Assistant Research Professor, (colabian@umich.edu), Institute for Social Research, University of Michigan, 426 Thompson Street, Room 2346, Ann Arbor, Ml 48106

The Youth, Education, and Society (YES) project (part of a larger research initiative, entitled Bridging the Gap: Research Informing Policy and Practice for Healthy Youth Behavior) is funded by the Robert Wood Johnson Foundation. The Monitoring the Future study is funded by the National Institute on Drug Abuse (DA001411). 
among secondary school students. ${ }^{10}$ Breaks are the main focus of this study because of limited recess in secondary school settings. In 2 intervention studies in middle schools, physical activity levels were significantly higher in the treatment condition that included physical activity breaks; however, the effects of breaks could not be isolated from other intervention components. ${ }^{11,12}$ In 2006, approximately two thirds of middle and one fifth of high schools in the United States had regular physical activity breaks during the school day outside of PE class and recess. ${ }^{8}$ As of the 2010-2011 school year, few school districts had strong policies in place to encourage opportunities for physical activity throughout the school day ( $11 \%$ of middle and $12 \%$ of high schools). ${ }^{13}$

The physical school environment also appears to influence how active students are at school, although most studies have focused on elementary schools. ${ }^{7,14}$ Higher numbers of indoor and outdoor school facilities (eg, basketball courts and gymnasiums) have been associated with higher in-school physical activity among secondary school students. ${ }^{7,15-17}$ However, 3 studies did not find similar associations with overall physical activity. ${ }^{18-20}$ The effect of school facilities on in-school but not overall physical activity levels is similar to findings for PE classes. ${ }^{21}$ Because there is little evidence that students compensate for increased activity levels at school by decreasing their activity at other times, ${ }^{22}$ the increased in-school activity may not be substantial enough to significantly affect overall physical activity.

This study builds on existing literature regarding physical activity breaks and school facilities in secondary schools. In particular, it adds to limited research on physical activity breaks in secondary schools and mixed findings regarding school facilities and overall physical activity levels. First, prevalence was estimated using nationally representative data from secondary schools in the United States. Second, school characteristics associated with having physical activity breaks or facilities were examined. Third, associations with student outcomes (overweight/obesity and physical activity) were examined.

\section{METHODS}

\section{Participants}

This report uses data from 2 studies, Monitoring the Future (MTF) and Youth, Education, and Society (YES), collected annually during 2008-2009 to 20112012 school years.

MTF design and methods. Monitoring the Future study design and methods have been described in detail elsewhere. ${ }^{23}$ Briefly, schools within primary geographic sampling units were selected with probability proportionate to size to obtain separate samples representative of 8 th, 10 th, and 12 th grade students in the United States. Replacement schools were selected using the same design if a selected school declined. Each school was invited to participate for 2 years. An original or replacement school was obtained for $97 \%$ of sample units, resulting in an average of 395 schools per year. Approximately 46,000 students responded each year, with student response rates averaging $90 \%, 87 \%$, and $83 \%$ for 8 th, 10 th, and 12 th graders, respectively.

YES design and methods. Each year, approximately 200 public and private schools were invited to participate in the YES study at the end of their second and final year of MTF participation (referred to as the MTF sample). To supplement the MTF sample of schools, a second nationally representative sample of approximately 600 public schools was surveyed each year (referred to as the supplemental sample). Supplemental schools were selected using a sampling procedure similar to MTF based on a rotating panel design; each school was invited to participate for three consecutive years, completing a new survey each year. ${ }^{24}$ Nonresponding schools were replaced using the same sampling procedure. No students were surveyed in the supplemental sample.

\section{Instruments}

School administrator survey. Survey questions were developed based on existing surveys and pilottested with a convenience sample of middle and high school administrators. Although formal reliability and validity studies were not conducted, respondents did not report difficulties answering survey questions.

School administrators reported the number of students enrolled in the school (school size; coded as $\leq 500$, 501-1000, or $>1000$ ), percentage of students eligible for free or reduced-price lunch (FRPL), and racial/ethnic composition of students. Free or reduced-price lunch was a proxy for school-level socioeconomic status (SES) and was categorized as $<15 \%$ (high-SES), $15-39 \%$ (medium-SES), and $\geq 40 \%$ (low-SES). Race/ethnicity was categorized as predominantly white $(\geq 66 \%$ white/non-Latino), majority black (>50\% black), majority Latino (>50\% Latino), and other (no racial/ethnic majority or a racial/ethnic majority other than white, black, or Latino). Region of the country (Northeast, Midwest, South, or West) and urbanicity (urban, suburban, or rural) were obtained from sample frame data.

Breaks were defined by an affirmative response to the following question: "Some schools offer activity breaks during school hours. Does your school provide (target grade) students opportunities to be physically active during the school day, other than in PE?" (yes or no). Unlike a previous study, ${ }^{8}$ this question did not exclude recess because recess is uncommon in middle and high schools.

Administrators reported which indoor or outdoor facilities were available to students. Indoor facilities 
were listed and included gymnasium(s) (courts for basketball, volleyball, etc.); indoor track(s); indoor pool(s); weight room; cardiovascular fitness center; wrestling room; dance studio; racquetball, squash, or handball court(s); or other indoor exercise or sports facilities. Outdoor facilities included outdoor tracks for walking, jogging, or running; outdoor pool(s); outdoor volleyball court(s); outdoor basketball court(s); outdoor tennis court(s); baseball or softball field(s); football or soccer field(s); general use field(s); or other outdoor exercise or sports facilities. Continuous measures of indoor and outdoor facilities were not ordinally associated with student outcomes in preliminary analyses; therefore, they were dichotomized based on the median for grade level (high vs low).

MTF student survey. Students self-reported sex, race/ethnicity, parental education, and household composition. Students were coded as non-Latino white, non-Latino black, Latino, or other race. Parental education was a proxy for SES and was dichotomized as at least 1 parent having at least some college education (high-SES) versus not (low-SES). Students also reported if they lived with both parents (yes vs no).

Students self-reported their height (in feet and inches) and weight (in pounds) using close-ended response categories. Body mass index (BMI) was calculated by dividing weight (in kilograms) by height (in meters) squared. Age- and sex-specific growth curves produced by the Centers for Disease Control and Prevention were used to determine whether each student was categorized as overweight $(\geq 85$ th BMI percentile) or obese ( $\geq 95$ th BMI percentile). ${ }^{25}$ Several studies have reported acceptable correlations between objectively measured and self-reported height and weight among adolescents when the purpose is to identify associations between variables. ${ }^{26,27}$

Moderate-to-vigorous intensity physical activity (MVPA) was assessed by asking students: "During the last 7 days, on how many days were you physically active for a total of at least 60 minutes/day? (Add up the time you spent in any kind of physical activity that increased your heart rate and made you get out of breath some of the time)." Responses were dichotomized into $\geq 5$ days/week versus $<5$ days. This question was originally developed as part of a 2-item clinical screening tool that has shown good reliability and moderate validity among middle and high school students. ${ }^{28,29}$

\section{Procedure}

Students in the MTF sample completed written questionnaires administered by study personnel during a normal class period. To minimize respondent burden, multiple versions of the questionnaire were created that included different subsets of items; each form was administered to a random subsample of students.
Questionnaires were mailed in the spring to school administrators in MTF and supplemental samples; a modest monetary incentive was used and follow-up calls and additional mailings were made as necessary to encourage participation. The same survey items were used with both samples. Respondents were instructed to answer for the current (just ending) school year. Most questions focused on the target grade. During the study period, 811 middle (8th grade) and 962 high (10th and 12th grade) schools participated with a total of 2746 observations due to repeated measurements in the supplemental sample. The combined response rate for both samples was $73 \%$ without replacement and $87 \%$ with replacement.

\section{Data Analysis}

In all analyses, 10th and 12th grades were combined (high school) because environmental characteristics such as school facilities would be expected to influence the entire high school.

School-level analyses. School-level analyses were conducted using combined data from public MTF and supplemental schools. Weights were used to adjust for sampling probabilities and the number of students in the target grade. Thus, school-level descriptive estimates represent the proportion or mean of target grade students in schools with specified characteristics rather than the proportion or mean of schools. Changes over time (modeled as categorical variable) and school characteristics associated with physical activity breaks or facilities were tested using generalized estimating equations to account for repeated measures in supplemental sample schools. ${ }^{30}$ The binomial distribution and logit link were used for dichotomous dependent variables. Type III significance tests were used to determine if each categorical independent variable was significant before examining pairwise comparisons; significant differences between categories were identified based on nonoverlapping 95\% CIs.

School-to-student analyses. Analyses of student outcomes used only the MTF sample because the supplemental sample did not have corresponding student-level data. All analyses adjusted for the sampling design and weights accounted for selection probabilities. Generalized estimating equation with binomial distribution and logit link for dichotomous outcomes were used to examine associations between physical activity breaks or facilities (school-level) and student-level measures while controlling for school- and student-level demographic characteristics and accounting for clustering by school. Generalized estimating equation estimates are interpreted as population-averaged, which is appropriate for the research questions. ${ }^{31}$ Grade 12 was a dummy variable in high school analyses because grades 10 and 12 
were combined. Interactions were tested to determine whether student sex, student-level SES, or school-level SES moderated associations between physical activity breaks or facilities and student measures. Because 3 interaction terms were tested in each model, the alpha criterion for significance was set at $0.05 / 3=0.017$ for the interaction terms. Analyses were conducted using SAS 9.2 (SAS Institute, Inc, Cary, NC).

\section{RESULTS}

\section{School Level: Prevalence of Physical Activity Breaks and Facilities}

More than one third of middle school students $(39.6 \%)$ attended schools that offered physical activity breaks, but breaks were less common among high school students (12.9\%). In contrast, high school students had more access to indoor and outdoor facilities compared with middle school students (3.0 vs 2.0 for indoor and 5.0 vs 4.0 for outdoor, respectively). Time trends were not statistically significant for physical activity breaks or facilities for middle or high school students (Table 1). Among middle and high school students, respectively, the most common indoor facilities were gymnasiums $(90.7 \%$ and $94.2 \%)$, weight rooms $(52.3 \%$ and $92.9 \%)$, and wrestling rooms $(18.9 \%$ and $56.5 \%)$, whereas the most common outdoor facilities were soccer/football fields $(79.2 \%$ and $88.6 \%)$, baseball/softball fields $(72.3 \%$ and $88.3 \%)$, and running tracks $(63.3 \%$ and $86.0 \%)$.

\section{School Level: School Characteristics Associated With Physical Activity Breaks and Facilities}

After adjusting for school-level characteristics, region and school size were most commonly associated with physical activity breaks and facilities in middle and high schools (Table 2). In middle schools, physical activity breaks were more common in the Northeast and West compared with the South and in the West compared with the Midwest, but there were no statistically significant regional differences in high schools. In middle schools, high indoor facilities were less common in the West than in the South. For middle and high schools, high outdoor facilities were more common in the West compared with all other regions except the Northeast for high schools.

Small middle schools were more likely to have physical activity breaks than medium or large ones. Large schools were more likely than small ones to have high indoor facilities (high schools only) and outdoor facilities (middle and high schools). Among high schools, large schools also had more indoor facilities than medium schools.

There were also some differences in physical activity facilities by race/ethnicity and school SES (FRPL eligibility) (Table 2). Among middle schools, lowSES schools, and those with majority black students
Table 1. Prevalence of Physical Activity Breaks and Facilities by Grade Level

\begin{tabular}{|c|c|c|c|c|c|c|c|c|}
\hline & \multicolumn{2}{|c|}{2009} & \multicolumn{2}{|r|}{2010} & \multicolumn{2}{|r|}{2011} & \multicolumn{2}{|r|}{2012} \\
\hline & & $\%$ & & $\%$ & & $\%$ & & $\%$ \\
\hline & $\mathbf{N}$ & Median & $\mathrm{N}$ & & $\mathbf{N}$ & & $\mathbf{N}$ & \\
\hline \multicolumn{9}{|c|}{ Physical activity breaks (\%) } \\
\hline 8th grade & $50^{*}$ & $35.9 \%$ & 339 & $39.4 \%$ & 321 & $38.2 \%$ & 314 & $41.9 \%$ \\
\hline 10th/12th grade & $91^{*}$ & $12.5 \%$ & 362 & $13.5 \%$ & 343 & $13.8 \%$ & 351 & $11.5 \%$ \\
\hline \multicolumn{9}{|c|}{ Number of indoor facilities ${ }^{\dagger}$ (median) } \\
\hline 8th grade & 327 & 2.0 & 338 & 2.0 & 320 & 2.0 & 314 & 2.0 \\
\hline 10th/12th grade & 377 & 3.0 & 363 & 3.0 & 344 & 3.0 & 351 & 3.0 \\
\hline \multicolumn{9}{|c|}{ Number of outdoor facilities ${ }^{\dagger}$ (median) } \\
\hline 8th grade & 328 & 4.0 & 339 & 4.0 & 321 & 4.0 & 314 & 4.0 \\
\hline 10th/12th grade & 377 & 5.0 & 363 & 5.0 & 343 & 5.0 & 351 & 5.0 \\
\hline
\end{tabular}

MTF, Monitoring the Future.

There were no significant time trends.

*In 2009, schools from the supplemental sample were not asked about physical activity breaks, so the sample size includes schools from only the MTF sample. ${ }^{\dagger}$ Range: 0-9.

were less likely to have high outdoor facilities than schools with higher SES students or other racial/ethnic compositions, respectively. High indoor facilities were less common among low-SES compared with higherSES high schools.

Suburban high schools had higher odds of high outdoor facilities compared with urban ones (adjusted odds ratio $[\mathrm{AOR}]=1.72,95 \%$ confidence interval [CI]: 1.21-2.44). No other significant associations with urbanicity were found in multivariate models.

\section{School-to-Student Results}

During the study period, complete data were available for models predicting overweight/obesity from 18,121 middle and 25,218 high school students and for models predicting MVPA from 5859 middle and 8218 high school students. A few associations between physical activity breaks or facilities, and studentlevel overweight/obesity were significant. Among middle school students, having physical activity breaks was associated with lower odds of overweight and obesity (Table 3). Also among middle schools, high indoor facilities were associated with lower odds of overweight among low-SES schools, lower odds of obesity among low-SES schools and students, and higher odds of obesity among medium-SES students (Table 4). Among high schools, high indoor facilities were associated with lower odds of obesity among girls but not boys (Table 4 ).

There were also significant associations between school facilities and student MVPA but only in high schools. High school students in schools with a high number of indoor facilities were more likely to have regular MVPA (Table 3). High outdoor facilities were also associated with higher odds of MVPA among high school students (Table 3) but were only significant among girls (Table 4). 


\begin{tabular}{|c|c|c|c|c|c|c|}
\hline \multirow[t]{2}{*}{ School Characteristic } & \multicolumn{2}{|c|}{ Physical Activity Breaks } & \multicolumn{2}{|c|}{ High*Indoor Facilities } & \multicolumn{2}{|c|}{ High*Outdoor Facilities } \\
\hline & $\mathrm{AOR}^{\dagger}(95 \% \mathrm{Cl})$ & $p^{\ddagger}$ & $\operatorname{AOR}^{\dagger}(95 \% \mathrm{Cl})$ & $p^{\ddagger}$ & $\operatorname{AOR}^{\dagger}(95 \% \mathrm{Cl})$ & $p^{\ddagger}$ \\
\hline 8th grade & $N=1023$ & & $N=1298$ & & $N=1301$ & \\
\hline Region & & $<.001$ & & .01 & & $<.001$ \\
\hline South & Ref & & Ref & & Ref & \\
\hline Midwest & $1.53(0.99-2.36)$ & & $1.10(0.72-1.68)$ & & $0.98(0.65-1.49)$ & \\
\hline Northeast & $1.81(1.11-2.97)$ & & $0.76(0.48-1.19)$ & & $0.74(0.46-1.18)$ & \\
\hline West & $4.08(2.59-6.43)$ & & $\mathbf{0 . 5 2}(0.35-0.79)$ & & $2.96(1.84-4.77)$ & \\
\hline School size $e^{\S}$ & & $<.001$ & & .002 & & $<.001$ \\
\hline$\leq 500$ & Ref & & Ref & & Ref & \\
\hline $501-1000$ & $\mathbf{0 . 4 1}(0.29-0.59)$ & & $0.77(0.56-1.07)$ & & $1.31(0.93-1.84)$ & \\
\hline$>1000$ & $0.42(0.27-0.67)$ & & $1.39(0.91-2.13)$ & & $2.50(1.56-4.02)$ & \\
\hline Race/ethnicity & & .11 & & .16 & & .01 \\
\hline$\geq 66 \%$ white & Ref & & Ref & & Ref & \\
\hline$>50 \%$ black & $0.47(0.24-0.91)$ & & $0.59(0.34-1.02)$ & & $0.42(0.24-0.74)$ & \\
\hline > 50\% Latino & $0.62(0.35-1.11)$ & & $0.74(0.47-1.16)$ & & $0.67(0.40-1.11)$ & \\
\hline Other & $0.81(0.53-1.24)$ & & $0.95(0.65-1.39)$ & & $0.92(0.63-1.33)$ & \\
\hline FRPL eligibility & & .06 & & .52 & & .004 \\
\hline$<15 \%$ & Ref & & Ref & & Ref & \\
\hline $15-39 \%$ & $0.59(0.35-0.99)$ & & $1.00(0.67-1.48)$ & & 0.87 (0.55-1.38) & \\
\hline$\geq 40 \%$ & $0.85(0.48-1.50)$ & & $0.82(0.52-1.29)$ & & $\mathbf{0 . 4 9}(0.30-0.81)$ & \\
\hline 10th/12th grade & $N=1145$ & & $N=1432$ & & $N=1431$ & \\
\hline Region & & .14 & & $<.001$ & & $<.001$ \\
\hline South & Ref & & Ref & & Ref & \\
\hline Midwest & $1.74(0.99-3.09)$ & & $4.78(3.24-7.03)$ & & $1.37(0.94-2.00)$ & \\
\hline Northeast & $1.06(0.50-2.26)$ & & $2.64(1.68-4.13)$ & & $1.67(1.08-2.58)$ & \\
\hline West & $1.66(0.92-2.99)$ & & $\mathbf{2 . 3 8}(1.59-3.57)$ & & $2.99(2.03-4.42)$ & \\
\hline School size $e^{\S}$ & & .24 & & $<.001$ & & $<.001$ \\
\hline$\leq 500$ & Ref & & Ref & & Ref & \\
\hline$\overline{501-1000}$ & $0.70(0.38-1.29)$ & & $2.05(1.21-3.46)$ & & $2.10(1.40-3.17)$ & \\
\hline$>1000$ & 0.61 (0.36-1.04) & & $8.35(5.15-13.54)$ & & $\mathbf{6 . 4 3}(4.27-9.71)$ & \\
\hline Race/ethnicity & & .17 & & .11 & & .11 \\
\hline$\geq 66 \%$ white & Ref & & Ref & & Ref & \\
\hline$>50 \%$ black & $0.60(0.22-1.67)$ & & $1.17(0.65-2.11)$ & & $0.54(0.31-0.95)$ & \\
\hline > 50\% Latino & $1.58(0.73-3.42)$ & & $1.62(0.91-2.89)$ & & $1.02(0.61-1.70)$ & \\
\hline Other & $1.44(0.82-2.52)$ & & $1.53(1.07-2.20)$ & & $0.78(0.54-1.13)$ & \\
\hline FRPL eligibility & & .59 & & .01 & & .21 \\
\hline$<15 \%$ & Ref & & Ref & & Ref & \\
\hline $15-39 \%$ & $0.74(0.38-1.41)$ & & $0.91(0.60-1.38)$ & & $0.94(0.60-1.46)$ & \\
\hline$\geq 40 \%$ & $0.85(0.41-1.76)$ & & $\mathbf{0 . 5 5}(0.35-0.87)$ & & $0.73(0.45-1.17)$ & \\
\hline
\end{tabular}

AOR, adjusted odds ratio; $\mathrm{Cl}$, confidence interval; FRPL, free or reduced-price lunch; Ref, reference category.

Statistically significant results $(\mathrm{p}<.05)$ are bolded to facilitate interpretation.

* "High" defined based on the median number of facilities for grade level.

${ }^{\dagger}$ Adjusted for year, school size, geographic region, urbanicity, race/ethnicity, and free or reduced-price lunch eligibility.

\#Type III p-value for school characteristic.

$\S$ Number of students enrolled in the school.

\section{DISCUSSION}

This is one of the first studies to examine school physical activity breaks and facilities, including associations with student-level measures, using nationally representative data. Compared with the 2006 School Health Policies and Programs Study (SHPPS), ${ }^{8}$ the prevalence of physical activity breaks in this study (school years 2008-2009 to 2011-2012) was much lower $(66.8 \%$ vs $39.6 \%$ and $22.2 \%$ vs $12.9 \%$ for middle and high schools, respectively) even though the SHPPS item explicitly excluded recess whereas this study did not. Prevalence may have declined since 2006 due to increasing emphasis on standardized tests. Alternatively, the difference could be due to data collection methods. Data were collected by computer-assisted personal interviews in SHPPS $^{8}$ and self-completed mailed questionnaires in this study. In SHPPS, PE staff was typically designated as respondents for PE modules ${ }^{8}$ compared with school administrators in this study. Both of these differences could have increased social desirability responding in SHPPS. Conversely, school administrators may be less aware of opportunities for students to be active throughout the day. Regardless of these differences, physical activity breaks were much less common among high schools than middle schools in both studies. Especially 
Table 3. Main Effects of Physical Activity Breaks and School Facilities on Student Outcomes by Grade Level, $2009-2012$

\begin{tabular}{|c|c|c|c|c|c|c|c|}
\hline & & $\% *$ & $\begin{array}{c}\operatorname{AOR}^{\dagger}(95 \% \mathrm{CI}) \\
\text { Overweight/Obese }\end{array}$ & $\% *$ & $\begin{array}{c}\operatorname{AOR}^{\dagger}(95 \% \mathrm{Cl}) \\
\text { Obese }^{\ddagger}\end{array}$ & \%* & $\begin{array}{c}\operatorname{AOR}^{\dagger}(95 \% \mathrm{CI}) \\
\text { Regular } \\
\text { Moderate-to-Vigorous } \mathrm{PA}^{\S}\end{array}$ \\
\hline \multicolumn{8}{|l|}{ 8th grade } \\
\hline \multirow[t]{2}{*}{ Physical activity breaks } & Yes & 25.9 & $\mathbf{0 . 9 1}(0.83-1.00)$ & 11.3 & $\mathbf{0 . 8 6}(0.75-0.99)$ & 57.1 & $1.11(0.92-1.33)$ \\
\hline & No & 27.4 & Ref & 12.7 & Ref & 54.4 & Ref \\
\hline \multirow[t]{2}{*}{ High"l indoor facilities } & Yes & 26.5 & $0.97(0.88-1.06)^{\natural}$ & 11.8 & $0.94(0.82-1.07)^{9}$ & 57.1 & $1.20(0.99-1.45)$ \\
\hline & No & 27.1 & Ref & 12.2 & Ref & 54.0 & Ref \\
\hline \multirow[t]{2}{*}{ High"l outdoor facilities } & Yes & 25.9 & $1.03(0.93-1.13)$ & 11.7 & $1.04(0.92-1.17)$ & 57.6 & $1.09(0.93-1.28)$ \\
\hline & No & 27.9 & Ref & 12.5 & Ref & 53.0 & Ref \\
\hline \multicolumn{8}{|l|}{ 10th/12th grade } \\
\hline \multirow[t]{2}{*}{ Physical activity breaks } & Yes & 27.9 & $1.02(0.90-1.15)$ & 12.5 & $1.03(0.86-1.24)$ & 50.8 & $1.10(0.89-1.37)$ \\
\hline & No & 27.2 & Ref & 12.2 & Ref & 48.8 & Ref \\
\hline \multirow[t]{2}{*}{ High"l indoor facilities } & Yes & 25.7 & $0.95(0.87-1.04)$ & 11.3 & $0.94(0.84-1.05)^{n}$ & 51.1 & 1.19 (1.03-1.38) \\
\hline & No & 28.6 & Ref & 13.1 & Ref & 47.4 & Ref \\
\hline \multirow[t]{2}{*}{ High"l outdoor facilities } & Yes & 25.9 & $0.99(0.91-1.08)$ & 11.3 & $0.92(0.82-1.04)$ & 50.7 & $1.22(1.06-1.39)^{9}$ \\
\hline & No & 29.1 & Ref & 13.5 & Ref & 46.8 & Ref \\
\hline
\end{tabular}

AOR, adjusted odds ratio; BMI, body mass index; Cl, confidence interval; MVPA, moderate-to-vigorous intensity physical activity; PA, physical activity; Ref, reference category. Statistically significant results $(\mathrm{p}<.05)$ are bolded to facilitate interpretation.

*The percentage of students with the outcome (eg, overweight, MVPA) in schools with ("Yes") and without ("No") the environmental characteristic.

${ }^{\dagger}$ Adjusted for year; student-level sex, race/ethnicity, parental education, living with both parents; and school-level school size, geographic region, urbanicity, race/ethnicity, and free or reduced-price lunch eligibility.

${ }^{\ddagger}$ Overweight/obese: $\mathrm{BMI} \geq 85$ th percentile for age/sex; obese: $\mathrm{BMI} \geq 95$ th percentile for age/sex.

$\S$ Defined as being physically active ("any kind of physical activity that increased your heart rate and made you get out of breath some of the time") for at least 60 minutes/day on $\geq 5$ of the last 7 days.

"IHigh" defined based on the median number of facilities for grade level.

"Main effects should be interpreted in the context of significant interaction effects, which are presented in Table 4.

Table 4. Effects of Physical Activity Breaks and School Facilities on Student Outcomes for Associations With Significant*Interaction Effects by Grade Level, 2009-2012

\begin{tabular}{|c|c|c|c|c|c|c|}
\hline Grade & School Variable & Student Measure & Moderator & Categories & $\mathrm{AOR}^{\dagger}$ & $(95 \% \mathrm{Cl})$ \\
\hline \multirow[t]{3}{*}{ 8th } & \multirow[t]{3}{*}{ High ${ }^{\ddagger}$ indoor facilities } & \multirow[t]{3}{*}{ Overweight/obese ${ }^{\S}$} & \multirow[t]{3}{*}{ School SESll } & Low & 0.83 & $(0.74-0.92)$ \\
\hline & & & & Medium & 1.26 & $(1.07-1.48)$ \\
\hline & & & & High & 1.14 & $(0.90-1.44)$ \\
\hline \multirow[t]{2}{*}{ 8th } & \multirow[t]{2}{*}{ High ${ }^{\ddagger}$ indoor facilities } & \multirow[t]{2}{*}{ Obese $^{\S}$} & \multirow[t]{2}{*}{ Student SES } & Low & 0.75 & $(0.62-0.92)$ \\
\hline & & & & High & 1.05 & $(0.89-1.23)$ \\
\hline \multirow[t]{3}{*}{ 8th } & \multirow[t]{3}{*}{ High ${ }^{\ddagger}$ indoor facilities } & \multirow[t]{3}{*}{ Obese $^{\S}$} & \multirow[t]{3}{*}{ School SES } & Low & 0.81 & $(0.68-0.95)$ \\
\hline & & & & Medium & 1.23 & $(0.98-1.54)$ \\
\hline & & & & High & 1.20 & $(0.84-1.71)$ \\
\hline \multirow[t]{2}{*}{ 10th/12th } & \multirow[t]{2}{*}{ High ${ }^{\ddagger}$ indoor facilities } & \multirow[t]{2}{*}{ Obese ${ }^{\S}$} & \multirow[t]{2}{*}{ Sex } & Female & 0.80 & $(0.68-0.93)$ \\
\hline & & & & Male & 1.04 & $(0.91-1.19)$ \\
\hline \multirow[t]{2}{*}{ 10th/12th } & \multirow[t]{2}{*}{ High ${ }^{\ddagger}$ outdoor facilities } & \multirow[t]{2}{*}{ MVPA $^{\#}$} & \multirow[t]{2}{*}{ Sex } & Female & 1.42 & $(1.19-1.70)$ \\
\hline & & & & Male & 1.04 & $(0.88-1.23)$ \\
\hline
\end{tabular}

AOR, adjusted odds ratio; $\mathrm{Cl}$, confidence interval; BMI, body mass index; MVPA, moderate-to-vigorous physical activity; SES, socioeconomic status.

Statistically significant results $(\mathrm{p}<.05)$ are bolded to facilitate interpretation.

*Because 3 interaction effects were tested in each model, alpha was set at 0.05/3=0.017.

${ }^{\dagger}$ Adjusted for year; student-level sex, race/ethnicity, parental education, living with both parents; and school-level school size, geographic region, urbanicity, race/ethnicity, and free or reduced-price lunch eligibility.

¥ “High" defined based on the median number of facilities for grade level.

$\S$ Overweight/obese: $\mathrm{BMI} \geq 85$ th percentile for age/sex; obese: $\mathrm{BMI} \geq 95$ th percentile for age/sex.

"Defined as follows based on percentage of students eligible for free and reduced-price lunch (FRPL): low ( $\geq 40 \% \mathrm{FRPL}$ ); medium (15-39\% FRPL); and high ( $<15 \% \mathrm{FRPL}$ ).

" "High" defined as one or both parents had more education than high school graduation and "low" as no parent had more than high school graduation, regardless of whether the student lived with 1 or both parents.

\#Defined as being physically active ("any kind of physical activity that increased your heart rate and made you get out of breath some of the time") for at least 60 minutes/day on $\geq 5$ of the last 7 days.

because few wellness policies at the school, district, or state levels currently include strong recommendations to provide opportunities for students to be active throughout the day, school administrators and partners should consider adding this component in future policy revisions. Future studies should also examine the frequency and quality of physical activity breaks, which may have stronger associations with studentlevel outcomes than the presence or absence of breaks.

To our knowledge, this is the first study to show significant associations between having school physical activity facilities (indoor or outdoor) and overall 
physical activity levels not limited to the school day. Three previous studies failed to find an association with overall physical activity. ${ }^{18-20}$ These studies used the same or similar measures as this study. Differences in student age may be responsible for some of the discrepant findings; 1 previous study only included students 15 years and younger ${ }^{19}$ and associations were only seen among high school students in this study. There may also be cultural or climatic differences between study populations because 2 previous studies were conducted in Scotland ${ }^{19}$ and Ontario. ${ }^{18}$

Although the magnitude of the associations between facilities and physical activity were small in this study, the public health impact could be large if all schools in the country had more facilities. ${ }^{32}$ Additionally, future studies should examine whether existing facilities are actually available to students throughout the day. To date, studies have focused on whether existing school facilities are open to other (nonschool) individuals or organizations outside of schools hours (joint-use agreements). ${ }^{33}$ School wellness policies could also require that physical activity facilities be available to students throughout the day, especially during noncurricular times such as lunch or recess.

If school facilities are associated with higher physical activity levels, smaller schools and those with higher percentages of low-SES and racial/ethnic minority students may need resources to expand school facilities. Because indoor facilities were associated with lower overweight and obesity only among lowSES schools and students, this type of intervention has the potential to reduce well-documented SES disparities in overweight. ${ }^{34}$ One previous study did not find a significant interaction between SES and effects of facilities on physical activity at school among 8th grade Norwegian students, but only 3\% of the sample was defined as "low-SES." 35 It is logical that school physical activity facilities could especially benefit lower-SES students because they likely have less access to similar facilities in community or commercial settings. ${ }^{36}$

Surprisingly, higher facilities were associated with higher overweight among medium- and high-SES middle schools, although the association was not significant for high SES schools. It is possible that higher SES schools have the financial means to add facilities in response to high rates of overweight and over time the facilities may contribute to decreased overweight. Longitudinal research is needed to examine this hypothesis and more research is needed to determine whether all SES-related findings are consistent in other studies.

Another interesting finding was that physical activity breaks in middle schools were associated with lower rates of obesity and overweight. Surprisingly, the logical complementary associations between breaks and physical activity behaviors were not found. Although they could not isolate the effects of physical activity breaks from other intervention components, 2 previous intervention studies in middle schools found significant effects of promoting physical activity throughout the school day on physical activity levels measured by observations or accelerometers. ${ }^{1,12}$ The self-reported physical activity measures used in this study may be less valid for smaller episodes of activity interspersed throughout the day. ${ }^{37}$ Future intervention studies should attempt to determine the extent to which physical activity breaks (as opposed to other intervention components) contribute to changes in objectively measured physical activity among middle and high school students.

\section{Limitations}

Although this study has numerous strengths, there are several limitations as well. Because data were observational and cross-sectional, causal inferences cannot be made. Data were self-reported by school administrators or students. Administrators were promised confidentiality to minimize social desirability bias. Study staff made follow-up phone calls to maximize the accuracy and completeness of administrators' responses. Self-reported physical activity levels are consistently higher than actual activity levels among adolescents. ${ }^{38}$ Additionally, weight is underreported and height is overreported, resulting in lower estimates of overweight or obesity compared with objective measures. ${ }^{39,26}$ For physical activity, there is little evidence that the validity of self-reported physical activity varies by demographic characteristics or weight status. ${ }^{28,40}$ Misreporting of weight and/or height is more common among females and those with higher BMI, but differences by other demographic characteristics (eg, race/ethnicity) have been inconsistent. ${ }^{39}$ Thus, due to a lack of consistent associations with student-level variables that could be associated with school physical activity policies and practices (eg, race/ethnicity), using self-reported as opposed to objective measures should not bias associations between school-level characteristics and overweight/obesity or MVPA.

\section{Conclusion}

Schools are increasingly encouraged to provide opportunities for physical activity outside of PE classes. Indoor school facilities may be particularly important for addressing student activity and overweight or obesity. Physical activity breaks also appear promising for middle school students but are uncommon in high schools. Students in all schools should have equal access to these resources and they may particularly benefit lower-SES students. 


\section{IMPLICATIONS FOR SCHOOL HEALTH}

School health staff can encourage school administrators to maintain existing or consider adding new physical activity facilities when possible, and ensure that students have time during the school day to use the facilities. School administrators, staff, and other school partners can consider including physical activity opportunities throughout the school day and access to existing physical activity facilities in school-, district-, and state-level wellness policies.

\section{Human Subjects Approval Statement}

Approval for the MTF study was obtained from the University of Michigan Behavioral Sciences Institutional Review Board (IRB). The IRB determined that the YES study was not research involving human subjects.

\section{REFERENCES}

1. Wechsler H, Devereaux R, Davis M, Collins J. Using the school environment to promote physical activity and healthy eating. Prev Med. 2000;31 (2, Pt 2):S121-S137.

2. Physical Activity Guidelines Advisory Committee. 2008 Physical Activity Guidelines for Americans. Washington, DC: US Department of Health and Human Services; 2008.

3. Committee on Prevention of Obesity in Children and Youth. Preventing Childhood Obesity: Health in the Balance. Washington, DC: National Academies Press; 2005.

4. Kahn EB, Ramsey LT, Brownson RC, et al. The effectiveness of interventions to increase physical activity: a systematic review. Am J Prev Med. 2002;22 (suppl 4):73-107.

5. Jago R, Baranowski T. Non-curricular approaches for increasing physical activity in youth: a review. Prev Med. 2004;39(1):157163.

6. Dobbins M, De Corby K, Robeson P, Husson H, Tirilis D. Schoolbased physical activity programs for promoting physical activity and fitness in children and adolescents aged 6-18. Cochrane Database Syst Rev. 2009;CD007651.

7. Ridgers ND, Salmon J, Parrish A, Stanley RM, Okely AD. Physical activity during school recess: a systematic review. Am J Prev Med. 2012;43(3):320-328.

8. Lee SM, Burgeson CR, Fulton JE, Spain CG. Physical education and physical activity: results from the School Health Policies and Programs Study 2006. J Sch Health. 2007;77(8):435-463.

9. Katz DL, Cushman D, Reynolds J, et al. Putting physical activity where it fits in the school day: preliminary results of the $\mathrm{ABC}$ (activity bursts in the classroom) for Fitness Program. Prev Chronic Dis. 2010;7(4):A82.

10. Parrish A, Okely AD, Stanley RM, Ridgers ND. The effect of school recess interventions on physical activity: a systematic review. Sports Med. 2013;43(4):287-299.

11. Sallis J, McKenzie T, Conway T, et al. Environmental interventions for eating and physical activity - a randomized controlled trial in middle schools. Am J Prev Med. 2003;24(3):209-217.

12. Haerens L, De Bourdeaudhuij I, Maes L, Cardon G, Deforche B. School-based randomized controlled trial of a physical activity intervention among adolescents. $J$ Adolesc Health. 2007;40(3):258-265.

13. Chriqui JF, Resnick EA, Schneider L, et al. School District Wellness Policies: Evaluating Progress and Potential for Improving Children's Health Five Years After the Federal Mandate. School Years 2006-07 Through 2010-11. Chicago, IL: Bridging the Gap
Program, Health Policy Center, Institute for Health Research and Policy, University of Illinois at Chicago; 2013. Available at: www.bridgingthegapresearch.org. Accessed July 1, 2013.

14. Harrison F, Jones AP. A framework for understanding school based physical environmental influences on childhood obesity. Health Place. 2012;18(3):639-648.

15. Haug E, Torsheim T, Sallis JF, Samdal O. The characteristics of the outdoor school environment associated with physical activity. Health Educ Res. 2010;25(2):248-256.

16. Sallis J, Conway T, Prochaska J, McKenzie T, Marshall S, Brown M. The association of school environments with youth physical activity. Am J Public Health. 2001;91 (4):618-620.

17. Nichol ME, Pickett W, Janssen I. Associations between school recreational environments and physical activity. J Sch Health. 2009;79(6):247-254.

18. Hobin E, Leatherdale S, Manske S, Dubin J, Elliott S, Veugelers P. A multilevel examination of factors of the school environment and time spent in moderate to vigorous physical activity among a sample of secondary school students in grades 9-12 in Ontario, Canada. Int J Public Health. 2012;57(4):699-709.

19. Kirby J, Levin KA, Inchley J. Associations between the school environment and adolescent girls' physical activity. Health Educ Res. 2012;27(1):101-114.

20. Durant N, Harris SK, Doyle S, et al. Relation of school environment and policy to adolescent physical activity. $J$ Sch Health. 2009;79(4):153-159.

21. Slingerland $M$, Borghouts L. Direct and indirect influence of physical education-based interventions on physical activity: a review. J Phys Act Health. 2011;8(6):866-878.

22. Baggett CD, Stevens J, Catellier DJ, et al. Compensation or displacement of physical activity in middle-school girls: the Trial of Activity for Adolescent Girls. Int J Obes. 2010;34(7):11931199.

23. Bachman JG, Johnston LD, O'Malley PM, Schulenberg JE. The Monitoring the Future Project After Thirty-Seven Years: Design and Procedures. Ann Arbor, MI: Institute for Social Research, University of Michigan; 2011:76.

24. Johnston LD, O'Malley PM, Terry-McElrath YM, FreedmanDoan P, Brenner JS. School Policies and Practices to Improve Health and Prevent Obesity: National Secondary School Survey Results, School Years 2006-07 and 2007-08. Ann Arbor, MI: Bridging the Gap Program, Survey Research Center, Institute for Social Research; 2011. Available at: www.bridgingth egapresearch.org/research/secondary_school_survey. Accessed September 27, 2012.

25. Kuczmarski RJ, Ogden CL, Guo SS, et al. 2000 CDC Growth Charts for the United States: methods and development. National Center for Health Statistics. Vital Health Stat. 2002;11(246):1-190.

26. Brener ND, Mcmanus T, Galuska DA, Lowry R, Wechsler H. Reliability and validity of self-reported height and weight among high school students. J Adolesc Health. 2003;32(4):281-287.

27. Goodman E, Hinden BR, Khandelwal S. Accuracy of teen and parental reports of obesity and body mass index. Pediatrics. 2000;106(1, Pt 1):52-58.

28. Ridgers ND, Timperio A, Crawford D, Salmon J. Validity of a brief self-report instrument for assessing compliance with physical activity guidelines amongst adolescents. J Sci Med Sport. 2012;15(2):136-141.

29. Prochaska J, Sallis J, Long B. A physical activity screening measure for use with adolescents in primary care. Arch Pediatr Adolesc Med. 2001;155(5):554-559.

30. Twisk JWR. Applied Longitudinal Data Analysis for Epidemiology. Cambridge, UK: Cambridge University Press; 2003.

31. Hubbard A, Ahern J, Fleischer N, et al. To GEE or not to GEE: comparing population average and mixed models for estimating the associations between neighborhood risk factors and health. Epidemiology. 2010;21(4):467-474. 
32. Jilcott S, Ammerman A, Sommers J, Glasgow RE. Applying the RE-AIM framework to assess the public health impact of policy change. Ann Behav Med. 2007;34(2):105-114.

33. Chriqui JF, Schermbeck R, Slater SJ, Schneider L, Barker DC, Chaloupka FJ. Joint Use Agreements: Creating Opportunities for Physical Activity - A BTG Research Brief. Chicago, IL: Bridging the Gap Program, Health Policy Center, Institute for Health Research and Policy, University of Illinois at Chicago; 2012. Available at: www.bridgingthegapresearch.org. Accessed January 4, 2013.

34. Delva J, O'Malley PA, Johnston LD. Racial/ethnic and socioeconomic status differences in overweight and healthrelated behaviors among American students: national trends 1986-2003. J Adolesc Health. 2006;39(4):536-545.

35. Haug E, Torsheim T, Samdal O. Physical environmental characteristics and individual interests as correlates of physical activity in Norwegian secondary schools: the health behaviour in school-aged children study. Int $J$ Behav Nutr Phys Act. 2008;5:47.
36. Powell LM, Slater S, Chaloupka FJ, Harper D. Availability of physical activity-related facilities and neighborhood demographic and socioeconomic characteristics: a national study. Am J Public Health. 2006;96(9):1676-1680.

37. Adamo KB, Prince SA, Tricco AC, Connor-Gorber S, Tremblay $\mathrm{M}$. A comparison of indirect versus direct measures for assessing physical activity in the pediatric population: a systematic review. Int J Pediatr Obes. 2009;4(1):2-27.

38. Helmerhorst HJF, Brage S, Warren J, Besson H, Ekelund U. A systematic review of reliability and objective criterion-related validity of physical activity questionnaires. Int J Behav Nutr Phys Act. 2012;9:103.

39. Sherry B, Jefferds ME, Grummer-Strawn M. Accuracy of adolescent self-report of height and weight in assessing overweight status. Arch Pediatr Adolesc Med. 2007;161(12):11541161.

40. LeBlanc AGW, Janssen I. Difference between self-reported and accelerometer measured moderate-to-vigorous physical activity in youth. Pediatr Exerc Sci. 2010;22(4):523-534. 